\title{
Based on the Health Index Model to Research the Discipline Ranking and Discipline Construction Policy
}

\author{
Baohuan Zhou \\ School of Public Affairs, University of Science and Technology of China, \\ 96 Jinzhai Road, Baohe District, Hefei 230026, China
}

\begin{abstract}
Construction of the world first-class university and the first-class discipline, is a major strategic decision made by the CPC central committee and the state council, in this environment, study of the healthy discipline development in colleges and universities has a certain practical significance. This article with the healthy development of discipline as the research object, based on the InCites and ESI database, to construct First-level discipline evaluation index system, using PCA method determine the index weight in the DHI (Discipline health index) model, establishing the healthy development of discipline empirical formula ,Dividing the discipline health level standard. Researches show that: (1) Top 100 colleges and universities in the QS chemistry discipline ranking, The health level Presents pyramid shape, about $32 \%$ of the college distribution within the partition between 0-60 partition;(2)In the health level rating, American colleges and universities keep in the top three, the average score of the three institutions discipline health index is up to 96.17 points;(3)Some universities ranking in DHI model change is bigger, we need to strengthen exchanges and cooperation between the advantages and disadvantages of discipline in the colleges and universities.
\end{abstract}

Keywords: Discipline ranking, PCA, DHI model,Health index, Policy analysis

DOI: $10.7176 /$ PPAR/9-3-02

Publication date:March $31^{\text {st }} 2019$

\section{Introduction}

On October 24, 2015, the State Council announced the Overall Plan for Coordinating the Advancement of World-Class Universities and First-Class Disciplines, clearly stating that the construction of a number of worldclass universities and first-class disciplines was accelerated and The country's"double-class" construction has also begun. "Double-class" construction is another national strategy after China's "211 Project" and "985 Project" in the field of higher education. With the announcement of the list of double-class universities in 2017 , the construction of "double-class" has become the hotspot of today's education. The focus of the discussion. The discipline is the cell of the university, and the world-class discipline is the foundation for building a world-class university.

The discipline is the cell of the university, and the world-class discipline is the foundation for building a world-class university(Zhou Guangli et al.2016). Disciplinary construction is the core of college construction. The healthy level of discipline development determines the overall strength and academic reputation of colleges and universities. Therefore, it is necessary to use discipline construction as a link to enhance the university's core competitiveness. At present, many scholars at home and abroad are studying the development model of university discipline construction, and most of them put forward the transition from scale development to connotation development, from the development of weight injection to the improvement of quality. At the same time, along with the development of network technology, the public data reflecting the quality of university disciplines is increasing rapidly. The research and development and use of relevant literature databases also provide a basis for the quality of in-depth monitoring disciplines, making some index systems and research methods driven by data structures in disciplines. Widely used in rankings and assessments.

In the discipline evaluation and model fitting, the index system construction and weight assignment are the key points. Which index classification criteria and weight calculation methods are selected are the differences of scholars.In the research on the evaluation method of index classification criteria, Li Xia et al. (2016) constructed a first-level discipline evaluation model based on SPCA (diluted principal component analysis). Zhu Meng et al.(2013) built a multi-level classification RD-DEA model based on DEA (data envelopment analysis) to solve the problem of multi-level index selection. In the research of index weight assignment method, Luo Jiaqi et al.(2015) used the expert interview, questionnaire, discussion and other forms, combined with scientific evaluation method AHP (Analytic Hierarchy Process), to quantitatively evaluate the performance of China's university science and technology innovation team. Liu Jinpei et al.(2017) proposed a new combination forecasting method for triangular fuzzy numbers of weight coefficients, and established a fuzzy variable weight combination forecasting model based on IOFWA (induced ordered fuzzy weighted average) operator.

In summary, most of the current research on the selection of indicators stays at the application level, and there are few theoretical studies. Based on the entropy theory, some literatures use the idea of entropy weighting(Anand P 1993) to quantify the index "discrimination", and construct an index screening model based 
on the combination of indicator importance and discrimination. In addition, when domestic and foreign scholars select evaluation indicators and establish index evaluation systems, they prefer to use the combination of global principal component analysis and DEA data envelopment analysis (Su Weihua et al. 2015), AHP analytic hierarchy and Defir method (Peng Zhanglin et al.2015), factor analysis and cluster analysis combined with the method(Su Weihua et al. 2012) and other methods of data processing methods.

Based on this background, the article attempts to extract key indicators based on the scientific evaluation method-principal component analysis method based on the scientific evaluation method in the form of factor analysis based on the in-depth understanding of the subject evaluation system and model construction, and assign weights to the university discipline development health index. Quantitative evaluation and model fitting. That is to say, based on the construction of the first-level discipline evaluation index system, establish the empirical formula of the discipline health development.

\section{Construction of discipline health index model}

\subsection{Basic connotation}

The discipline health index model is based on the discipline as the analysis object, based on the disciplinary evaluation index system, to test the empirical formula of the discipline development quality. At present, the diversified health index model research is to deepen the benchmarking practice in all walks of life in China. Its originality is to first display the complicated and diverse data and use an index to express it. It is a tool to improve health management. Secondly, the process statistics technology in process management is sublimated into a health management theory, in order to reach the benchmark value of the research target index as much as possible.

According to the product cycle theory proposed by Raymond Vernon (1966), the product life cycle is divided into three phases, namely, the new product phase, the mature product phase and the standard product phase, and the standard life cycle analysis considers Things need to go through four stages of development, growth, maturity and decline. They are projected into the process of university discipline development. They also have the characteristics of life cycle. From the stage of germination to maturity standardization, the process needs to go through the process from sub-health to health. Health index model As a quantitative indicator system for measuring different stages of the life cycle of a discipline, it is a complex organizational system.

\subsection{Setting principles of evaluation index system}

- targeted principles. Determine the corresponding indicators for the purpose and object of the evaluation, fully reflect the characteristics of the evaluation object, highlight the key points of the evaluation indicators, and reflect the uniqueness of the evaluation of the healthy development of the disciplines in universities.

- Practical principles. The indicator system should be simple and moderate, the evaluation method is simple and easy, and the relevant data is easy to collect.

- Scientific principles. The evaluation index system is the product of the combination of theory and practice. Through scientific and technical summarization of work rules, indicators at all levels must be independent and related at the same time, making the indicator system an organic whole (Xiong Qingnian et al.2017).

- System optimization principles. The number of indicators and the structure of the indicator system should conform to the systemic principle, that is, to reflect the content of the evaluation object more comprehensively and systematically with fewer indicators, to avoid duplication of indicators and structure is too cumbersome.

\subsection{Selection of evaluation indicators}

The process of discipline development includes two links: academic input and output. Academic input is the basis of discipline generation and development. Academic output is the expression of discipline maturity and standardization. This paper takes discipline as the object of analysis to explore the different degrees of discipline development health. Therefore, the construction of health index model mainly centers on the academic output of discipline and refers to the fourth round of discipline evaluation in China. According to the index categories of Incites and ESI databases, this paper describes the subject health index model from three levels, including the output of papers, scholars and international cooperation. That is to say, the organization system is mainly composed of three components.

The article constructs the evaluation index system of the healthy development degree of the discipline development in the three aspects of disciplinary influence, subject productivity and disciplinary development. According to the concept of subject value (Li Penghu 2017), the discipline influences the citation influence, the relative global average, and the cited percentage of the paper. The influences of journals' standardized citation influences and citation influences reflect the influence of disciplines in the development of disciplines in 
universities, and mainly reflect the research influence of disciplines and the level of discipline competitiveness among institutions.

The WOS (Web of Science) literature, the number of cited papers, the citation frequency and the $\mathrm{h}$ index are used as reflections of the subject productivity in the process of university discipline development, and represent the academic level of the discipline as a whole. The two indicators of the percentage of international cooperation papers and the percentage of horizontal cooperation papers are used as reflections of the development of disciplines in the process of university discipline development, and the level of discipline cooperation and publication is expressed.

\subsection{Determination of index weight}

\subsubsection{Establishment and Standardization of indicator matrix}

The article determines the index weight based on principal component analysis (Wang Xiaoying et al. 2013). It is assumed that there are $p$ indicators in the academic health model index system, and there are $n$ research objects, that is, there are $n$ objects, each of which has $\chi_{1}, \chi_{2}, \ldots, \chi_{p}$ elements are composed, and the corresponding element data are shown in the following table 1. The multi-indicator matrix $\chi_{n p}$ is obtained.

Table 1. Research object element data

\begin{tabular}{|c|cccccc|}
\hline \multirow{2}{*}{ Objects } & \multicolumn{1}{|c|}{ Elements } \\
\cline { 2 - 6 } & $\chi_{1}$ & $\chi_{2}$ & $\cdots$ & $\chi_{j}$ & $\cdots$ & $\chi_{p}$ \\
\hline 1 & $\chi_{11}$ & $\chi_{12}$ & $\cdots$ & $\chi_{1 j}$ & $\cdots$ & $\chi_{1 p}$ \\
2 & $\chi_{21}$ & $\chi_{22}$ & $\cdots$ & $\chi_{2 j}$ & $\cdots$ & $\chi_{2 p}$ \\
$\cdots$ & $\cdots$ & $\cdots$ & $\cdots$ & $\cdots$ & $\cdots$ & $\cdots$ \\
$i$ & $\chi_{i 1}$ & $\chi_{i 2}$ & $\cdots$ & $\chi_{i j}$ & $\cdots$ & $\chi_{i p}$ \\
$\cdots$ & $\cdots$ & $\cdots$ & $\cdots$ & $\cdots$ & $\cdots$ & $\cdots$ \\
$n$ & $\chi_{n 1}$ & $\chi_{n 2}$ & $\cdots$ & $\chi_{n j}$ & $\cdots$ & $\chi_{n p}$ \\
\hline
\end{tabular}

The original variables are $\chi_{1}, \chi_{2}, \ldots, \chi_{p}$, After the dimension reduction process, set their comprehensive indicators, that is, the new variables are $z_{1}, \mathrm{z}_{2}, \ldots, z_{p}(m \leqq p)$ then:

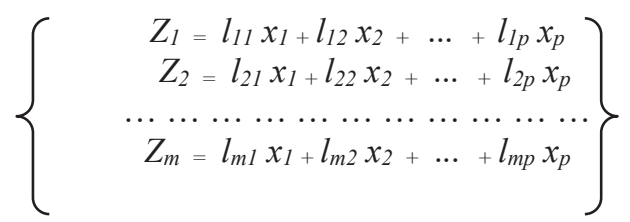

2.4.2 Correlation coefficient matrix and eigenvalue of normalized matrix $Z$

To find the principal component $Z_{i}$ is to determine the coefficient $j_{i j}$, which are the feature vectors corresponding to the $m$ larger eigenvalues of the correlation coefficient matrix of $\chi_{1}, \chi_{2}, \ldots, \chi_{p}$ respectively. Correlation coefficient calculation formula:

$$
r_{i j}=\frac{\sum_{k=1}^{n}\left(x_{\kappa i}-\overline{x_{i}}\right)\left(x_{\kappa j}-\overline{x_{j}}\right)}{\sqrt{\sum_{k=1}^{n}\left(x_{\kappa i}-\overline{x_{i}}\right)^{2} \sum_{k=1}^{n}\left(x_{\kappa j}-\overline{x_{j}}\right)^{2}}}
$$

According to the formula, the correlation coefficient matrix between the $p$ variables is:

$$
\mathscr{\aleph}=\left(\begin{array}{ccc}
r_{11} & \cdots & r_{1 p} \\
\vdots & \ddots & \vdots \\
r_{p 1} & \cdots & r_{p p}
\end{array}\right)
$$

Solution characteristic equation: $|\lambda E-g|=0$

Find the eigenvalue: $\lambda_{i}(i=1,2, \cdots, \mathrm{p})$

The $p$ eigenvalues are arranged in order of magnitude, which is: $\lambda_{1} \geq \lambda_{2} \geq \cdots \geq \lambda_{p} \geq 0$

Then press the formula: $\left|\lambda_{i} E-g_{n}\right| \varepsilon_{i}=0$

The eigenvectors $\epsilon_{i}(i=1,2, \cdots, \mathrm{p})$ corresponding to $\lambda_{i}$ are obtained respectively

2.4.3 Determine principal components and weights

The contribution rate of principal component $Z_{i}$ is:

$$
Q_{i}=\frac{\lambda_{i}}{\sum_{\mathrm{k}=1}^{\mathrm{p}} \lambda_{\mathrm{k}}}(i=1,2, \cdots, \mathrm{p})
$$


The cumulative contribution rate of the first $i$ principal component is:

$$
Q=\frac{\sum_{\mathrm{k}=1}^{i} \lambda_{\mathrm{k}}}{\sum_{\mathrm{k}=1}^{\mathrm{p}} \lambda_{\mathrm{k}}}(i=1,2, \cdots, \mathrm{p})
$$

The current cumulative contribution rate of $i$ principal components reaches $85 \%-95 \%$, and the first $i$ principal components are taken as new variables(I.T.Jolliffe 1986). That is $g_{i j}=\sqrt{\lambda_{i}} \epsilon_{i j}(i, j=1,2, \cdots, \mathrm{p})$, the load of the first i principal component on the original variable is obtained, as shown in the following table:

Table 2. Principal component loading matrix

\begin{tabular}{|c|c|c|c|c|}
\hline \multirow[t]{2}{*}{ Original variable $X_{i}$} & \multicolumn{4}{|c|}{ main ingredien } \\
\hline & $Z_{1}$ & $Z_{2}$ & $\cdots$ & $Z_{i}$ \\
\hline$x_{1}$ & $q_{11}$ & $q_{21}$ & $\cdots$ & $g_{i 1}$ \\
\hline $\begin{array}{l}x_{2} \\
\ldots\end{array}$ & $q_{12}$ & $q_{22}$ & $\cdots$ & $\xi_{i 2}$ \\
\hline$x_{n}$ & $\cdots$ & $\cdots$ & $\cdots$ & $\cdots$ \\
\hline & $\&_{1 p}$ & $\xi_{2 p}$ & $\cdots$ & $\xi_{i p}$ \\
\hline
\end{tabular}

According to the coefficient $j_{i j}$ of the principal component $Z_{i}$ corresponding to the original variable $x_{i}$, the coefficient of the corresponding index is calculated, that is, the weight assignment is as follows:

$$
x_{p} \text { Indicator coefficient }=\frac{\frac{\rho_{1 \mathrm{p}}}{\sqrt{\lambda_{1}}} * \mathcal{Q}_{1}+\frac{\rho_{2 \mathrm{p}}}{\sqrt{\lambda_{2}}} * \mathcal{Q}_{2}+\cdots+\frac{\rho_{i \mathrm{p}}}{\sqrt{\lambda_{i}}} * \mathcal{Q}_{i}}{\mathcal{Q}}
$$

\subsection{DHI model determination}

According to the above-mentioned index weight assignment, it is possible to develop a comprehensive health evaluation model for colleges and universities, and combine multiple evaluation index values into a comprehensive comprehensive evaluation value, and evaluate multiple subjects, namely, university chemistry subjects. The comprehensive evaluation subject health index model is as follows :

$$
D H I=\sum_{i}^{10} c_{i} w_{w i}
$$

$C_{i}$ is the assignment of the first $\mathrm{i}$ index in the evaluation system, and $W_{w i}$ is the composite weight of the first $\mathrm{i}$ index. When using this model to evaluate the health of university discipline development, the university chemistry disciplines involved in the evaluation are assigned to 10 integrated indicators according to the abovementioned index system, multiplied by the corresponding synthetic weights, and the scores of each index are obtained. Then the scores are summed up, and the scores are the health index of each university chemistry discipline, i.e. the level of the index. It reflects the comprehensive ability of chemistry in Colleges and universities.

\section{Empirical analysis}

\subsection{Data acquisition}

Sample selection: Based on InCites database, statistical period is 2008-2018, literature type is Article \& Review, ESI discipline classification criterion - direction of chemistry discipline is selected, and according to the Chemistry professional ranking of QS World University discipline ranking in 2018-2019, TOP100 chemical discipline ranking index data are selected as training samples, taking into account the different development courses of the discipline. According to the comprehensive score of QS Chemistry, there are 500 schools that pass the grade line, and then select the index data of 100 universities as test samples, ranking at 180-200; 280-320; $380-400 ; 480-500$, respectively. A total of 200 university institutions were selected, corresponding to 15 subindicators and 30,000 sample data.

\subsection{Data processing and calculation}

In this paper, SPSS22.0 data statistical analysis software is used to process the average value of 30,000 sample data, calculate the average value of 15 subdivision index data of various chemical disciplines for ten years, and do cross-sectional data analysis.

According to Kaiser's KMO metrics, the original variables are suitable for factor analysis. The Bartlett statistic is 2121.237, and the corresponding probability Sig value is $0.000<0.0001$. The correlation coefficient 
matrix is significantly different from the unit matrix. That is to say, the correlation matrix is not a unit matrix and can be used for factor analysis.

The reliability test is as follows:

\section{Table 3: Correlation Matrix}

\begin{tabular}{|c|c|c|c|c|c|c|}
\hline & & Zscore $(\mathbf{P M})$ & Zscore(CNCI) & Zscore(DC) & Zscore(IR) & Zscore $(\mathrm{CI})$ \\
\hline \multirow[t]{10}{*}{ Related } & Zscore(PM) & 1.000 & -.071 & -.367 & -.093 & -.131 \\
\hline & Zscore (CNCI) & -.071 & 1.000 & .600 & .993 & .981 \\
\hline & Zscore(DC) & -.367 & .600 & 1.000 & .616 & .618 \\
\hline & Zscore(IR) & -.093 & .993 & .616 & 1.000 & .990 \\
\hline & Zscore(CI) & -.131 & .981 & .618 & .990 & 1.000 \\
\hline & Zscore(CPM) & .998 & -.038 & -.323 & -.060 & -.098 \\
\hline & Zscore(JNCI) & .006 & .809 & .441 & .818 & .784 \\
\hline & Collaboration & -.436 & .070 & .312 & .084 & .083 \\
\hline & Top & -.006 & .918 & .527 & .928 & .900 \\
\hline & Cited & .765 & .526 & .139 & .518 & .480 \\
\hline & & Zscore(CPM) & Zscore(JNCI) & Collaboration & Top & Cited \\
\hline \multirow[t]{10}{*}{ Related } & Zscore(PM) & .998 & .006 & -.436 & -.006 & .765 \\
\hline & Zscore $(\mathrm{CNCI})$ & -.038 & .809 & .070 & .918 & .526 \\
\hline & Zscore(DC) & -.323 & .441 & .312 & .527 & .139 \\
\hline & Zscore(IR) & -.060 & .818 & .084 & .928 & .518 \\
\hline & Zscore(CI) & -.098 & .784 & .083 & .900 & .480 \\
\hline & Zscore(CPM) & 1.000 & .025 & -.423 & .023 & .792 \\
\hline & Zscore(JNCI) & .025 & 1.000 & .085 & .827 & .452 \\
\hline & Collaboration & -.423 & .085 & 1.000 & .000 & -.273 \\
\hline & Top & .023 & .827 & .000 & 1.000 & .562 \\
\hline & Cited & .792 & .452 & -.273 & .562 & 1.000 \\
\hline
\end{tabular}

The eigenvalues of the correlation coefficient matrix are shown in Table 4 . The cumulative contribution rate of the first three principal components is $90.938 \%$. Therefore, the three principal components $Z_{1}, Z_{2}$ and $Z_{3}$ can fully reflect the healthy development degree of the subjects in the 200 universities ranked by QS World University Chemistry Discipline Rank.

Table 4: Total Variance Explained

\begin{tabular}{|c|c|c|c|c|c|c|}
\hline \multirow{2}{*}{ component } & \multicolumn{3}{|c|}{ Initial Eigenvalue } & \multicolumn{3}{|c|}{ Rotating Square Sum Loading } \\
\cline { 2 - 7 } & Total & Variance \% & accumulate \% & Total & Variance \% & accumula \% \\
\hline 1 & 5.286 & 52.865 & 52.865 & 5.225 & 52.248 & 52.248 \\
2 & 3.032 & 30.315 & 83.180 & 2.762 & 27.616 & 79.864 \\
3 & .776 & 7.758 & 90.938 & 1.107 & 11.073 & 90.938 \\
4 & .499 & 4.991 & 95.929 & & & \\
5 & .255 & 2.546 & 98.475 & & & \\
6 & .105 & 1.049 & 99.524 & & & \\
7 & .028 & .284 & 99.807 & & & \\
8 & .016 & .160 & 99.968 & & & \\
9 & .003 & .029 & 99.997 & & & \\
10 & .000 & .003 & 100.000 & & & \\
\hline
\end{tabular}

The total variance explained in Table 4, i.e. the contribution rate of eigenvalues, shows that the contribution rates of the first, second and third principal components are $52.865 \%, 30.315 \%$ and $7.758 \%$ respectively, and the total contribution rate reaches $90.938 \%$. The weight of the second-level index to the upper level is calculated according to the load number of principal components corresponding to the index component matrix and the contribution rate of eigenvalues and variances of the principal components.

Above is the result of SPSS22.0 software data analysis. The gravel maps show the criteria for selecting principal components. The first and second principal components are selected when the eigenvalue is greater than 1. According to the composition maps of rotating space, the range and classification of each index can be clearly displayed.

The results of subject data processing are shown in Table 5: 
Table 5: Calculation result of Index data

\begin{tabular}{cccccc}
\hline index & $\begin{array}{c}\boldsymbol{Z}_{\mathbf{1}} \\
\text { coefficient }\end{array}$ & $\begin{array}{c}\boldsymbol{Z}_{\mathbf{2}} \\
\text { coefficie }\end{array}$ & $\begin{array}{c}\boldsymbol{Z}_{\mathbf{3}} \\
\text { coefficie }\end{array}$ & $\begin{array}{c}\text { Coefficients in the } \\
\text { integrated score model }\end{array}$ & Index Weight \\
\hline ZPM & -0.012 & 0.562 & 0.210 & 0.198515 & 0.101364 \\
ZCNCI & 0.426 & -0.020 & -0.068 & 0.235471 & 0.120233 \\
ZDC & 0.283 & -0.233 & 0.221 & 0.105761 & 0.054003 \\
ZIR & 0.429 & -0.032 & -0.066 & 0.233414 & 0.119183 \\
ZCI & 0.422 & -0.052 & -0.092 & 0.220188 & 0.11243 \\
ZCPM & 0.003 & 0.561 & 0.232 & 0.208631 & 0.106529 \\
ZJNCI & 0.375 & 0.013 & -0.011 & 0.221451 & 0.113075 \\
collaboration & 0.037 & -0.337 & 0.881 & -0.0158 & -0.00807 \\
Top & 0.413 & 0.026 & -0.114 & 0.238883 & 0.121976 \\
Cited & 0.251 & 0.444 & 0.215 & 0.311932 & 0.159275 \\
\hline
\end{tabular}

Because the sum of the weights of all indicators is 1, the weights of indicators need to be normalized on the basis of calculating the index coefficients in the comprehensive model. The comprehensive evaluation model of health index DHI, i.e. the empirical formula is as follows:

$\mathrm{DHI}=0.101 \mathrm{PM}+0.12 \mathrm{CNCI}+0.054 \mathrm{DC}+0.119 \mathrm{IR}+0.112 \mathrm{CI}+0.107 \mathrm{CPM}+0.113 \mathrm{JNCI}+0.122 \mathrm{Top}+0.159 \mathrm{Cited}-$ 0.008 Collaboration

\section{Main research conclusions}

\subsection{Analysis conclusion}

According to the empirical formula fitted by DHI model, the results of university subject health index are calculated, and the corresponding relationship of health index levels is divided. The nodes among the levels are selected as $0,60,70,80,90$ and 100 in turn, as shown in Table 6 :

Table 6: The level correspondence of health index

Index value range
$90-100$
$80-90$
$70-80$
$60-70$
$0-60$
Below 0

level
I
II
III
IV
V
VI

According to the empirical formula of DHI subject health index model, the organization score of TOP100 in QS Chemistry University Discipline ranking is calculated. The discipline health index shows obvious step difference. This paper chooses institutions with DHI score of 70 or more. According to cluster analysis, corresponding to the corresponding relationship of the health index level in Table 6 , it divides the discipline health level of institutions as shown in Table 7: 
Table 7: The institutional DHI level partition table:

\begin{tabular}{|c|c|c|}
\hline University & DHI Score & level \\
\hline $\begin{array}{c}\text { University of California Berkeley } \\
\text { Northwestern University } \\
\text { Stanford University }\end{array}$ & $\begin{array}{c}100 \\
97.23 \\
91.28\end{array}$ & I \\
\hline $\begin{array}{c}\text { Massachusetts Institute of Technology (MIT) } \\
\text { Ecole Polytechnique Federale de Lausanne } \\
\text { University of California Los Angeles } \\
\text { Nanyang Technological University } \\
\text { Harvard University } \\
\end{array}$ & $\begin{array}{l}85.96 \\
84.79 \\
84.57 \\
83.67 \\
82.37\end{array}$ & II \\
\hline $\begin{array}{c}\text { National University of Singapore } \\
\text { Georgia Institute of Technology } \\
\text { University of Texas Austin } \\
\text { University of Gottingen } \\
\text { University of Michigan } \\
\text { University of Chicago } \\
\text { Princeton University } \\
\text { University of Cambridge } \\
\text { California Institute of Technology } \\
\text { Tsinghua University } \\
\text { Zhejiang University } \\
\text { Carnegie Mellon University } \\
\text { University of California Santa Barbara } \\
\text { University of Science \& Technology of China }\end{array}$ & $\begin{array}{l}78.97 \\
78.84 \\
78.82 \\
78.53 \\
78.42 \\
78.28 \\
75.88 \\
74.35 \\
74.32 \\
74.27 \\
72.39 \\
72.37 \\
72.26 \\
70.29\end{array}$ & III \\
\hline
\end{tabular}

According to table 7, American universities rank the top three steadily, with an average score of 96.17 in each of the three institutions, while the five adjacent universities rank the lowest in the 80-90 sub-area, especially MIT (Massachusetts Institute of Technology) from the first place in QS ranking to the fourth place in DHI ranking, and five institutions rank the fourth in DHI ranking. The average score of the subject health index is only 84.27 , which is quite different from that of the top three institutions. The difference of the subject health level is obvious, and the health level is a pyramid.

The article divides the health index level into 6 levels. According to the DHI model, there are 3 colleges in the first level, 5 colleges in the second level, and 14 colleges in the third level. The total number of colleges in the first three ranks is only 22 . In the sample colleges and universities, the proportion of data only reaches $11 \%$. The divisional health level of top universities is too obvious. The degree of disciplinary development between universities is relatively large. In the low-end of the pyramid, between 0-60 partitions, the distribution is about With $32 \%$ of colleges and universities, it can be seen that there are obvious gaps between the institutions in terms of advantages and disadvantages, and exchanges and cooperation between universities are needed.

\subsection{Policy suggestion}

According to the comprehensive scores of the institutions in the QS World University Rankings, the article made a simple comparison of ranking changes. Since the QS World University Rankings uses six indexes to measure the world universities, the weights of their indicators are peer-reviewed in the academic field. (40\%), global employer evaluation (10\%), number of papers cited by unit teachers $(20 \%)$, teacher/student ratio $(20 \%)$, international student ratio (5\%), international teacher ratio $(5 \%)$, The article collects sample data according to the InCites database. The reputation index and the proportional data are subjectively defined values based on the feedback results of the questionnaire [16]. Therefore, the index selection excludes the type index, and the larger proportion is set on the cited frequency indicator. As a result, there is a certain change in the comprehensive score. Compared with the scores of the indicators with subjective estimates, the DHI scores of the articles are more comparable and scientific.

From the level correspondence table of the health index, it can be seen that the health index model fits the university subject ranking compared to the QS subject ranking, there are some discrepancies, which is the result of the different standards of the evaluation index system, MIT chemistry subject ranking From the first place in the QS discipline to the third place in the health index ranking, while the UCB (University of California, Berkeley) rose from the seventh to the first, from the perspective of the index system structure, mainly because of the disciplinary impact indicators. The weight of the upper assignment is relatively large, and the high-level papers account for a better fit of the health index.

In order to test whether the health index ranking of the model fitting is scientific, the article will rank the chemistry class index of the university chemistry subject to the ESI chemistry ranking, basically agree with the ESI institution ranking, exclude some types of non-Academic institutions, and find Northwestern University, 
China. Zhejiang University, Kyoto University of Japan, Nanyang Technological University of Singapore, etc. compared to the QS subject rankings significantly improved institutions.

Some universities in the DHI model have changed greatly. For example, Zhejiang University in China rose from the 99th in the QS discipline to the 19th, the University of Science and Technology of China rose from 53 to 22, and the Nanjing University from 67 to 27 . The proportion of domestic colleges and universities is generally increased in volume. In particular, Zhejiang University has a large volume of disciplines, which leads to a significant increase in rankings. The DHI model has a large weight value in highly cited literature, relative to foreign reputation. The index system with larger weights is more representative of the characteristics of domestic university discipline development, and the disciplinary health assessment model is more applicable.

\section{References}

Zhou Guangli., Wu Jianxin, J. (2016 ), "What is the World-Class Discipline”, China Higher Education Research, $65-73$.

Li Xia., Wang Xing., Ren Chao. \& Lin Mengquan, J. (2016), "Under the condition of complex data inquiry -based on the SPCA method for evaluating the level of discipline construction level discipline evaluation model analysis", Academic Degrees \& Graduate Education 12, 30-33.

Zhu Meng., Meng Wei., Yu Cheng. \& Liu Wenbin,J. (2013), "Evaluation of research efficiency of higher education institutions using DEA Models of multi-level classification", Science $R$ esearch Management 12, 221-22.

Luo Jiaqi., Kuang Haibo, J. (2015), "The performance evaluation index system of scientific research resources in universities'science and technology innovation team", Science Research Management 1, 116-121.

Liu Jinpei., Zhu Jiaming., Chen Huayou.\&Fang Qionghong, J. (2017), "Method Research on Fuzzy Variable Weights Combination Forecasting”, Statistics and Decision 16, 5-9.

Anand P, J (1993), "The Philosophy of Intransitive Preference", The Economic Journal 103, 337-346.

$\mathrm{Su}$ Weihua., Luo Gangfei.\&Zeng Shouzhen, J. (2015), "Efficiency evaluation of university research in Zhejiang Province", Science Research Management 9, 141-148.

Peng Zhanglin., Zhang Qiang.\&Yang Shanlin, J. (2015), "Overview of Comprehensive Evaluation Theory and Methodology", Chinese Journal of Management Science 11, 245-256.

$\mathrm{Su}$ Weihua, J. (2012), "The multi-index comprehensive evaluation technology and application research of review and understanding", statistical research 8, 98-107.

Raymond Vernon, J. (1966), "International Investment and International Trade in the Product Cycle ", The Quarterly Journal of Economics 5, 190-207.

Xiong Qingnian., Zhang Duanhong, J. (2017), "Concept and Classification: Value Assessment in the Subject Evaluation", Exploring Education Development 3, 1-7.

Li Penghu, J. (2017), "Basic Logic for Building World First-rate Universities", Journal Of Granduate Education 4, 77-81.

Wang Xiaoying., Zhou Baoping, J. (2013), "Mathematical modeling and mathematics experiment (second edition)", Science Press, 16-26.

I.T.Jolliffe, M. (1986), "Principal component analysis", Springer Verlag, 150-166.

Zhou Guangli.,Wu Jianxin, J. (2016), "What is the Global Standard for Academic Evaluation-Empirical analysis based on the four global university rankings", China Higher Education Research 4, 51-56. 